\title{
Multiple Gossypibomas Resulting in Intra-Abdominal Abscess and Intestinal Fistula after Caesarean Section: a Case Report
}

\author{
Jie Ding MD', Yu Xia MD², Zhong-min Zhang MD \\ ${ }^{1}$ Department of Gastrointestinal Surgery, Guizhou Provincial people's Hospital, Guiyang, China. \\ ${ }^{2}$ Department of Stomatology, Guizhou Provincial people's Hospital, Guiyang, China. \\ dingjieboy@126.com
}

\begin{abstract}
:
Background: Gossypiboma is a term for a surgical complications resulting from foreign materials, such as a surgical sponge, accidentally left inside a patient's body.

Methods: We report a rare case of woman who presented with multiple gossypibomas resulting in intra-abdominal abscess and intestinal fistula after caesarean section.

Results: A 35-year-old Chinese woman was referred to our hospital for abdominal pain of three months, diarrhea of one month and melena of one week. Three months ago, she had once undergone caesarean section at a county hospital. The abdominal computed tomography revealed two mass-like enhancement in right iliac fossa and left iliac fossa. Therefore, a laparotomy was performed. An operation including removal of intraabdominal foreign object, abdominal abscess drainage, partial enterectomy, partial sigmoid colon resection and descending colostomy were performed. The operation revealed that two gossypibomas in the abdomen led to intra-abdominal abscess and intestinal fistula.
\end{abstract}

Conclusion: Gossypiboma, although it's a rare postoperative complication, should be taken into consideration in the diagnosis of abdominal mass, especially in the patients who has undergone abdomenal operations.

Sub headings: Gossypibomas resulting in intra-abdominal abscess and fistula

Gossypiboma (cottonoid or textiloma1) is a term used to describe a foreign object, such as a mass of cotton matrix or a sponge, which is accidentally left in a body cavity during an operation [1]. It is an unacceptable and entirely preventable surgical complication. Open surgery and laparoscopic surgery are the common approach in the treatment of gossypiboma. However, endoscopy can also be feasible for both diagnosis and treatment of gossypiboma [2-6]. We report a case of multiple gossypibomas resulting in intra-abdominal abscess and intestinal fistula after caesarean operation, which was successfully treated by open operation.

\section{Case Presentation}

A 35-year-old Chinese woman was referred to our hospital for abdominal pain of three months, diarrhea of one month and melena of one week. Three months ago, she underwent caesarean section atacounty hospital. After operation, she always hadintermittent lower abdominal dull pain and distension, but had no history ofnausea, vomiting, poor appetite orbody weight loss. She once went to Guiyang Obstetric and Gynecology Hospital and The Third Guizhou Provincial People's Hospital, receivedanti-inflammatory treatment, she feeled the abdominal pain relieved to some extent. One 
Jie Ding et al.

week ago, these symptoms appeared again, accompanied with black tarry stools, 3-4 times a day. For further treatment, she was referred to our hospital.

Abdominal computed tomography (CT) scan showed two mass-like enhancement in right iliac fossa and left iliac fossa $(98 \mathrm{~mm} \times 68 \mathrm{~mm}$ and $102 \mathrm{~mm} \times 56 \mathrm{~mm}$ in size respectively), indicating the possibility of large iliac fossa abscesses (Fig.1 to Fig.2). Colonoscopy showed that colonic mucosa within $20 \mathrm{~cm}$ to $25 \mathrm{~cm}$ of the anal verge become swollen and red, a white gauze retention in the enteric cavity, causing intestine slightly narrower(Fig.3). Barium enema showed the possibilty of the fistula between Sigmoid colon and small intestine (Fig.4).
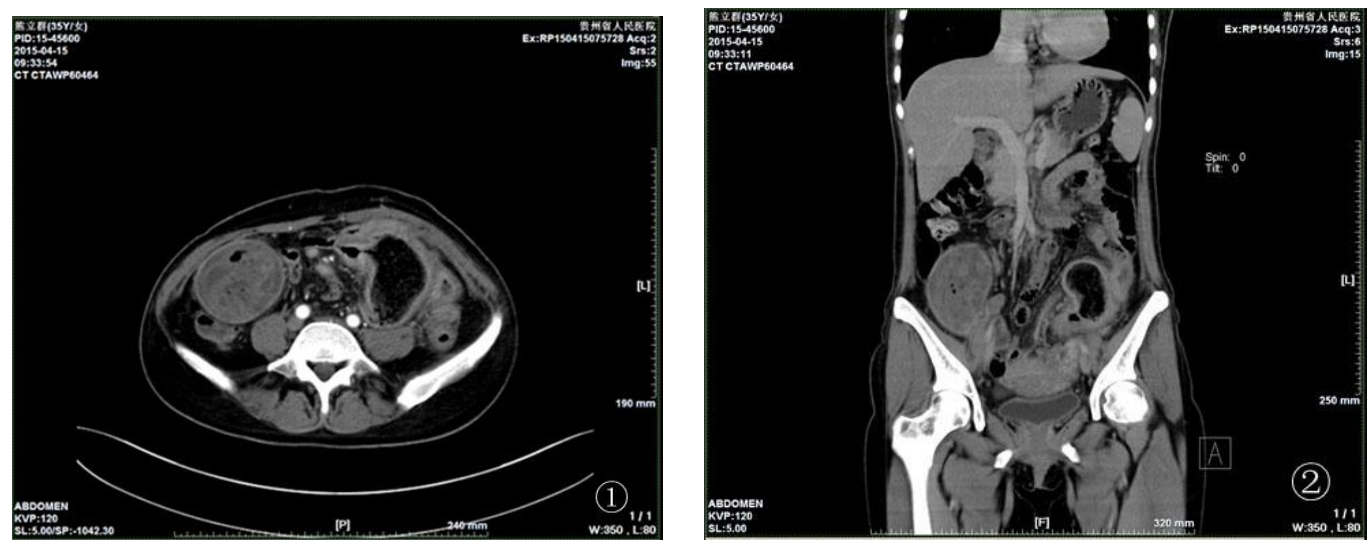

Fig. 1 and Fig. 2 CT image indicates that Two mass-like enhancement in right iliac fossa and left iliac fossa, $98 \mathrm{~mm} \times 68 \mathrm{~mm}$ and $102 \mathrm{~mm} \times 56 \mathrm{~mm}$ in size respectively, indicating the possibility of large iliac fossa abscesses

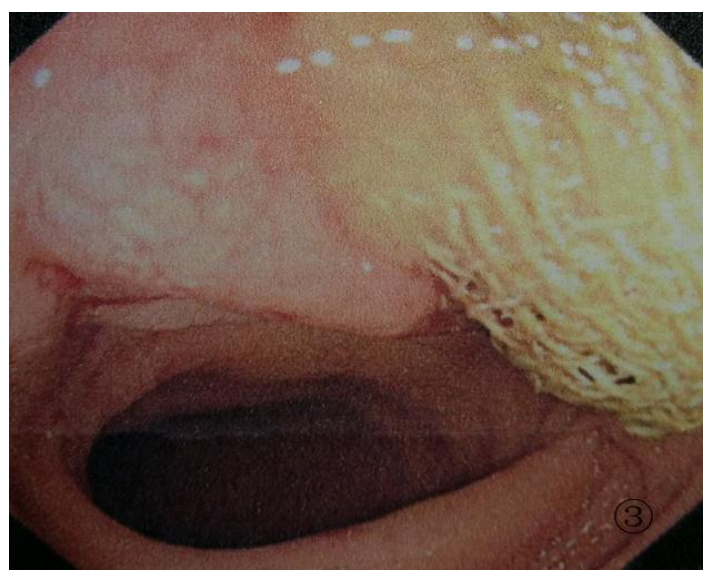

Fig3. Colonic mucosa within $20 \mathrm{~cm}$ to $25 \mathrm{~cm}$ of the anal verge become swollen and red, a white gauze retention in the enteric cavity, causing intestine slightly narrower

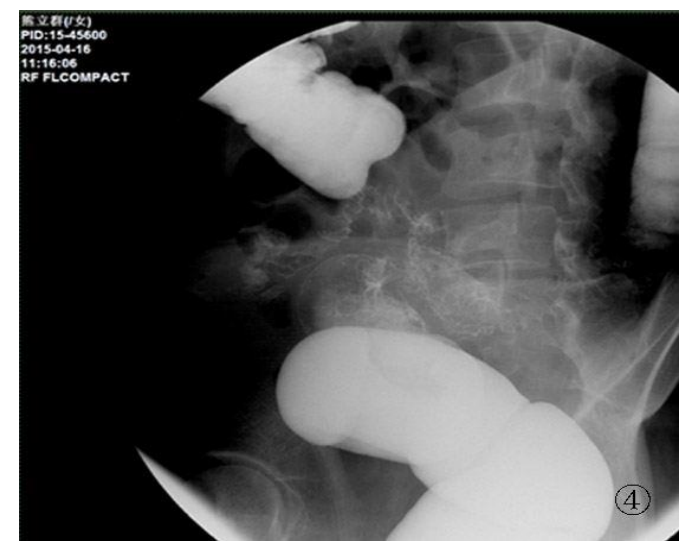

Fig4. Barium enema showed the possibilty of the fistula between Sigmoid colon and small intestine 


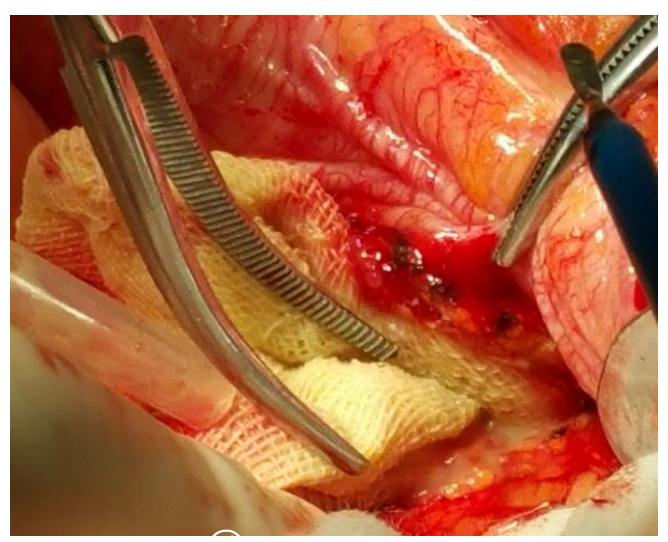

Fig5. Omentum adhered with right lower abdominal wall and terminal ileum, forming a big abscess, a gauze was found inside the vomica

After preoperative preparation, the patients have been implemented an operation including removal of intraabdominal foreign object, abdominal abscess drainage, partial enterectomy, partial sigmoid colon resection and descending colostomy. Laparotomy revealed omentum shifted to the right lower quadrant, adhered with right lower abdominal wall and terminal ileum, forming a big abscess, $12 \mathrm{~cm}$ $\times 10 \mathrm{~cm}$ in size. Cutting the abscess, a gauze was found inside the vomica, which was been takenout of abdominal cavity (Fig.5).Exposing the left lower quadrant, ileum, sigmoid colon and left lower quadrant were found to adhered with each other, and formed a abscess $(8 \mathrm{~cm} \times 10 \mathrm{~cm}$ in size $)$. Amazingly, there was also a gauze retained inside the vomica (Fig 6 to Fig 7). Part of the gauze migrated into sigmoid colon, taking out the gauze, multiple fistulas could seen between Sigmoid colon and small intestine (Fig.8).Following surgery, the patient made a good recovery.
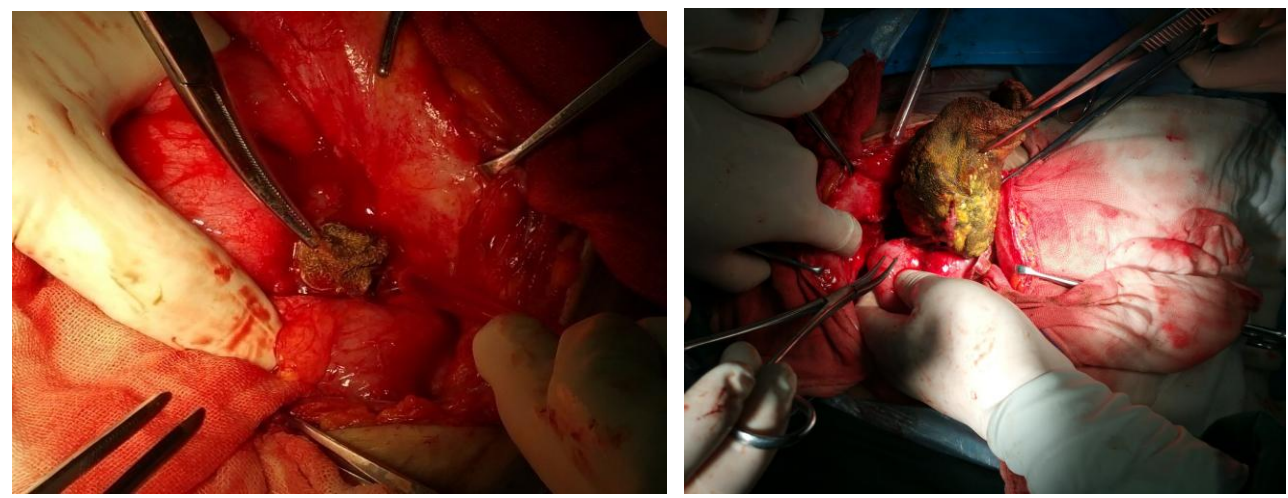

Fig6. and Fig7. Ileum, sigmoid colon and left lower quadrant were found to adhered with each other, and formed a abscess in the left lower quadrant. Also, there was also a gauze retained inside the vomica

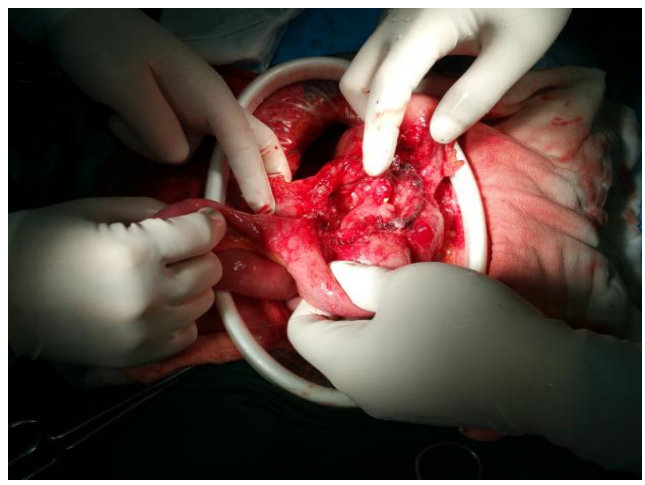

Fig8. Multiple fistulas could be found between Sigmoid colon and small intestine 


\section{DISCUSSION}

Retained surgical sponge can cause serious complications, which bring traumatic psychological and physical injury to the patients. A systematic literature review by Wan et al. identified 254 gossypiboma cases documented over the 45 years [7]. They noted that the gossypibomas were most commonly found in the abdomen (56\%), pelvis (18\%), and thorax (11\%). The most common detection methods were computed tomography (61\%), radiography (35\%), and ultrasound (34\%). Retained surgical sponge can be followed by adhesion, abscess, ileus, intestinal necrosis and fistula [7], operation should be performed to remove the lesions. The most common reason of gossypiboma in abdomen is cholecystectomy,caesarian section, hysterectomy and laparotomy [a].Many risk factors of retained surgical sponge were identified, the most important factors are emergency surgery and unplanned changes in the operation [9-11]. Pain, palpable mass, and fever were the leading signs and symptoms [12]. However, $6 \%$ of cases were asymptomatic. It is reported that a gauze was hidden in a male patients without any indisposed symptoms for 38 years, which was accidentally discovered in an operation due to a car accident [13]. The cases of retaining two gauzes in the body at the same time are very rare [14].

Retained surgical sponge belongs to unskillful and faulty medical treatment, which brings patients unnecessary harm, and easy to stimulate medical disputes. Therefore, checks in the operating room should be strictly implemented. Whenever engages in an operation in body cavity or deep tissue, all the number of instruments should be checked carefully before and after surgery. Medical safety must be firmly grasped, and alarm bells should sound over the trauma of remnants in peritoneal to patients.

\section{CONClusion}

Gossypiboma brings traumatic psychological and physical injury to the patients, checks in the operating room should be strictly implemented to avoid the incidence of retained surgical sponge in a body cavity.

\section{REFERENCES}

[1] Williams RG, Bragg DG, Nelson JA. Gossypiboma--the problem of the retained surgical sponge. Radiology 1978;129(2):323-6.

[2] Peyrin-Biroulet L, Oliver A, Bigard MA. Gossypiboma successfully removed by upper-GI endoscopy. Gastrointest Endosc. 2007;66(6):1251-2.

[3] Sozutek A1, Yormaz S, Kupeli H, Saban B. Transgastric migration of gossypiboma remedied with endoscopic removal: a case report. BMC Res Notes. 2013;6:413.

[4] Hinrichs C, Methratta S, Ybasco AC. Gossypiboma treated by colonoscopy. Pediatr Radiol. 2003;33(4):261-2.

[5] Erbay G, Koç Z, Calişkan K, Araz F, Ulusan S.Imaging and clinical findings of a gossypiboma migrated into the stomach.Turk J Gastroenterol. 2012;23(1):54-7.

[6] Ojha S, Gall T, Sodergren MH, Jiao LR. A case of gossypiboma mimicking intrahepatic cholangiocarcinoma. Ann R Coll Surg Engl. 2014 Oct;96(7):e14-6.

[7] Wan W, Le T, Riskin L, Macario A. Improving safety in the operating room: a systematic literature review of retained surgical sponges.Curr Opin Anaesthesiol. 2009;22(2):207-14.

[8] Lv YX, Yu CC1, Tung CF, Wu CC. Intractable duodenal ulcer caused by transmural migration of gossypiboma into the duodenum--a case report and literature review.BMC Surg. 2014;14:36.

[9] Erdil A, Kilciler G, Ates Y, Tuzun A, Gulsen M, Karaeren N, Dagalp K. Transgastric migration of retained intraabdominal surgical sponge: gossypiboma in the bulbus. Inter Med 2008, 47(7):613-615. 
[10] Dakubo J, Clegg-Lamptey J, Hodasi W, Obaka H, Toboh H, Asempa W:An intra-abdominal gossypiboma. Ghana Med J 2009, 43(1):43-45.

[11] Sumer A, Carparlar MA, Uslukaya O, Bayrak V, Kotan C, Kemik O, Llikerden U. Gossypiboma: retained surgical sponge after a gynecoogic procedure. Case Report Med 2010, 917626.

[12] Justo JW, Sandler P, Cavazzola LT. Retained surgical sponge mimicking GIST: Laparoscopic diagnosis and removal 34 years after original surgery.J Minim Access Surg. 2013;9(1):29-30.

[13] Peng m, Zou W. Retained surgical sponge in the abdomen for 38 years. Clinical Misdiagnosis \& Mistherapy. 2003, 16(3): 163.

[14] Karthik SM1, Kumar RM1, Ananthakrishnan N. Gossypiboma: an unusual foreign body of the male urethra.Indian J Surg. 2013; 75(1):77-78. 